\title{
Does a similar metabolic reprogramming occur in Fe-deficient plant cells and animal tumor cells?
}

\author{
Gianpiero Vigani* \\ Dipartimento di Produzione Vegetale, Università degli Studi di Milano, Milano, Italy \\ *Correspondence: gianpiero.vigani@unimi.it
}

Plant and animal cells are very different from each other in many of their functions. However, the comparison of their behavior during different stress conditions shows intriguing similarities. Interestingly, one of these cases is the comparison between the metabolic reprogramming in plant cells under Fe deficiency and in developing cancers in animal cells.

Recent reviews have explored the idea that Fe deficiency induces a reprogramming of metabolism in dicotyledonous plants (Vigani, 2012) in order to take up Fe from the soil. Briefly, under Fe-limited growth conditions, root cells reprogram their metabolism as follows:

1 An increase in glycolysis and an impairment in the respiratory chain. Fe is an essential cofactor for several mitochondrial enzymes and under Fe deficiency the functions of mitochondria are strongly impaired, without losing importance in regulating metabolic responses to this stress, and surprisingly, in Fe-deficient roots the number of mitochondria increases, even if they are less efficient. In this context, Fe-deficient cells activate glycolysis to supply energy for the Fe acquisition mechanism (Vigani, 2012, and references therein).

2 An increase in anaerobic reactions. López-Millán et al. $(2000,2009)$ reported that Fe deficiency increased activities of fermentative enzymes such as lactate dehydrogenase (LDH) and pyruvate decarboxylase (PDC), which could contribute to the oxidation of the pyridine nucleotide pool in tomato and sugar beet roots. These data were also supported by a microarray analysis of Fe-deficient Arabidopsis (Thimm et al., 2001) and by proteomic data obtained from sugar beet and cucumber roots, in which putative alcohol dehydrogenase and malate dehydrogenase proteins accumulated under Fe deficiency (Donnini et al., 2010; Rellán-Álvarez et al., 2010).

3 An increase in the oxidative pentose phosphate pathway (OPP). In particular, some cytosolic $\mathrm{NAD}(\mathrm{P})^{+}$-dependent dehydrogenases, such as glucose 6-phosphate dehydrogenase (oxidative pentose phosphate pathway; Vigani, 2012), showed an increased activity in order to produce the reducing equivalents necessary to sustain Fe uptake.

Surprisingly, a similar metabolic reprogramming occurs in animal tumor cells: their entire metabolism is reorganized in order to sustain cell growth and proliferation. Briefly, some cancer cells are characterized by the following metabolic adaptations:

1 A tumor-associated alteration is the "Warburg phenomenon," in which glucose is preferentially converted to lactate by enhancing glycolysis and fermentative reactions rather than completely oxidized by oxidative phosphorylation (OXOPHOS; Warburg et al., 1924; Warburg, 1956; BrahimiHorn et al., 2007). This metabolic alteration is therefore often referred to as "aerobic glycolysis" (Vander Heiden et al., 2009; Figure 1), being a fermentative pathway actually occurring in aerobic conditions. Further, as observed in Fe-deficient plant cells, some tumor cells contain abnormally high numbers of mitochondria (Carew and Huang, 2002).

2 Cancer cells generate carbonic and lactic acids, with lactate being the principal end product of aerobic glycolysis. Such acids favor tumor invasion (Swietach et al., 2007) and suppress anticancer immune effectors (Fischer et al., 2007). Lactate that is produced by tumor cells can be taken up by stromal cells to regenerate pyruvate that can then either be extruded to refuel the cancer cells or be used for the respiratory chain (Koukourakis et al., 2006).

3 Tumors can metabolize glucose through the OPP to generate NADPH that ensures the function of the cell's antioxidant defenses against a hostile microenvironment resulting in tolerance to chemotherapeutic agents (Gatenby and Gillies, 2004).

One of the mechanisms activating aerobic glycolysis involves the hypoxia-inducible factor (HIF), a transcription factor that is activated not only by hypoxic stress but also by oncogenic, inflammatory, metabolic, and oxidative stress (Harris, 2002; Semenza, 2007; Taylor and Pouyssegur, 2007). HIF-1 consists of a constitutively expressed subunit, HIF- $1 \beta$, and an oxygen-regulated transcriptional activator, HIF- $1 \alpha$. The stability and activity of the $\alpha$ subunit of the HIF are regulated by post-translational modifications (e.g., hydroxylation). In normoxia, hydroxylation of two proline residues and acetylation of a lysine residue in the oxygendependent degradation domain (ODDD) of HIF- $1 \alpha$ triggers its association with the pVHL E3 ligase complex, leading to HIF- $1 \alpha$ degradation via ubiquitin-proteasome pathways. The hydroxylation mechanism is mediated by a prolyl hydroxylase (PHD), which belongs to the 2-oxoglutarate-dependent dioxygenase family and catalyzes the formation of 4-hydroxyproline, requiring 2-oxoglutarate and $\mathrm{O}_{2}$ as cosubstrates, $\mathrm{Fe}^{2+}$ as a cofactor, and ascorbate for optimal activity. The accumulation of HIF- 1 has been found in various cancers. When for some reason the hydroxylationdependent degradation mechanism is not activated, HIF-1 accumulates and stimulates the conversion of glucose to pyruvate and lactate by upregulating glucose transporter isoform 1 (GLUT1), hexokinase (HK1 and HK2), and lactate dehydrogenase A (LDHA), as well as the lactate-extruding 


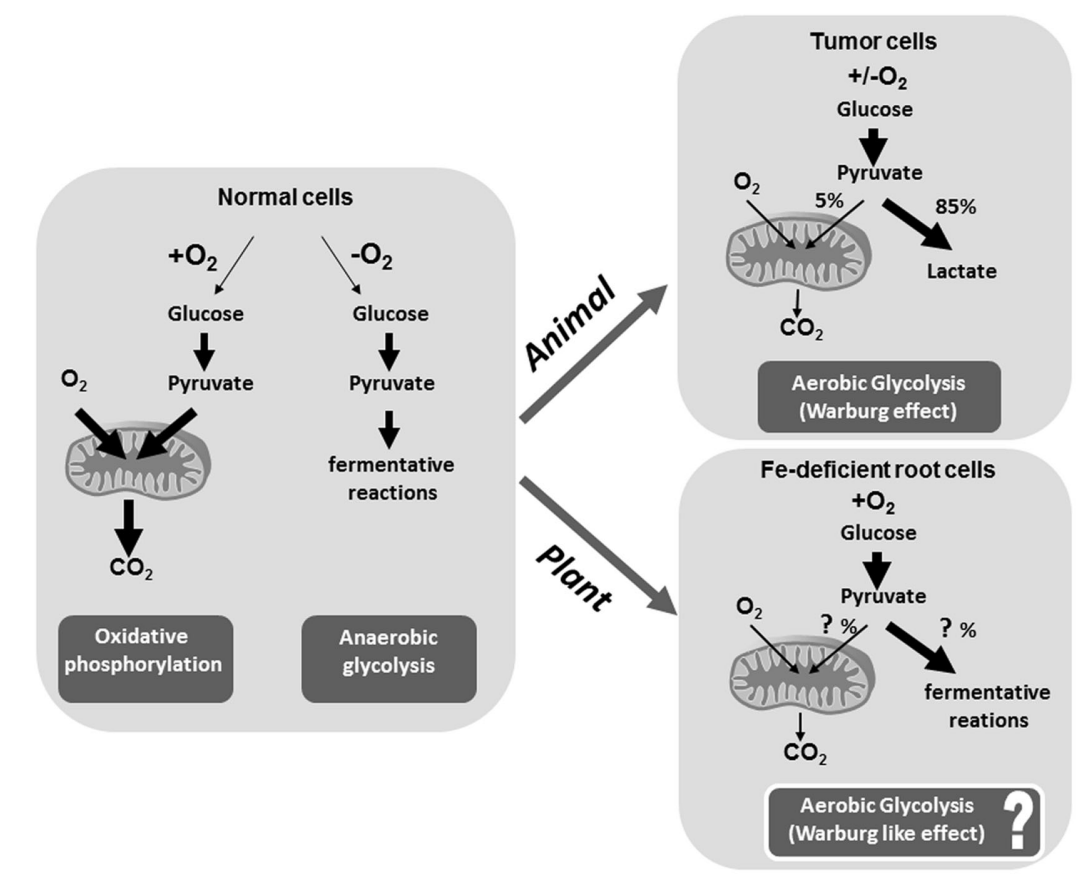

FIGURE 1 | Schematic representation of the differences between oxidative phosphorylation, anaerobic glycolysis, and aerobic glycolysis (Warburg effect) in animals and iron deficiency-induced metabolic responses in plant cells. In the presence of oxygen, normal cells (both animal and plant) first metabolize glucose to pyruvate via glycolysis and then completely oxidize most of that pyruvate in the mitochondria during oxidative phosphorylation (OXOPHOS). When oxygen is limiting, cells can redirect the pyruvate generated by glycolysis away from mitochondrial OXOPHOS by inducing a fermentative pathway (anaerobic glycolysis). The Warburg effect observed in cancer cells tend to convert most glucose to lactate regardless of whether oxygen is present or not (aerobic glycolysis). A similar Warburg effect could be observed also in Fe-deficient plant cell, in which the slowed OXOPHOS, because of the lack of Fe, determine the up-regulation of glycolysis-fermentative reactions. Since this metabolic switch occurred in the presence of oxygen, we can consider it as the induction of aerobic glycolysis. (The Figure is a adaptation/reinterpretation of Figure 2 reported in Vander Heiden et al., 2009). enzyme monocarboxylate transporter 4 (MCT4; Semenza, 2007). Apparently, the Warburg effect mediated by the induction of HIF-1 seem to be strictly related to hypoxia. Nevertheless, in tumor cells, it has been postulated that hypoxia represents an important aspect of cancer biology but just as a late-occurring event. Thus hypoxia may not be considered as a major contributor in the switch to aerobic glycolysis by cancer cells (Vander Heiden et al., 2009). Tumor cells develop the metabolic switch from an energetically more (oxidative phosphorylation) to a less (aerobic glycolysis) efficient metabolism because, being proliferating cells, they have important metabolic requirements that extend beyond a simple high ATP production. Indeed, during proliferation, cells must replicate all of their cellular contents: the glucose must be directed toward the synthesis of several cellular mol- ecules such as acetyl-CoA, glycolytic intermediates and ribose for the biosynthesis of fatty acids, non-essential amino acids, and nucleotides, respectively (Vander Heiden et al., 2009; Figure 1). OXOPHOS is not impaired but it is slowed down in order to sufficiently satisfy the energetic request for the life of the cell and to balance the ATP/ ADP ratio (Vander Heiden et al., 2009).

Moving the focus on to plants, it seems that the metabolic alteration induced under Fe deficiency mimics the metabolic alteration observed in the cancer cells, raising an intriguing hypothesis. Low Fe content causes a slowdown of OXOPHOS as well as an induction of glycolysis plus fermentative reactions even if in aerobic conditions. Since, as in tumor cells, also in Fe-deficient plant cells, glucose metabolism is mainly (not totally, the mitochondria being still working in both cases) directed toward glycolysis plus fermentative reactions, can we postulate a Warburg-like effect also in Fe-deficient plants? In tumor cells the induction of glycolysis toward fermentative reaction is referred to as aerobic glycolysis instead of anaerobic glycolysis, because it occurs independently of the presence of oxygen. Since in Fe-deficient plant cells the metabolic switch observed, which is similar to that of the animal tumor cells, is originally the consequence of the lack of Fe and not of the lack of oxygen, could it be considered as an aerobic glycolysis? (Figure 1). Furthermore, in Fe-deficient plant cells, the glucose directed toward an aerobic-like glycolysis process allows it to satisfy both (i) the energetic request for cell survival and (ii) the request of glycolytic intermediates for the synthesis of several metabolic compounds useful to the cell to overcome Fe deficiency, such as organic acids.

Despite the metabolic reprogramming of Fe-deficient plant cells showing similarities with that occurring in animal tumor cells, it is important to take into account some metabolic aspects in which these two systems may differ from each other. Although the Warburg effect is considered a hallmark of cancer research, it occurs in some specific tumor cells. Indeed some tumors contain two subpopulations of cancer cells that differ in their energy-generating pathways, but they seem to function symbiotically. One subpopulation consists of glucosedependent cells (Warburg effect displaying cells) that secrete lactate, whereas the cells belonging to the second subpopulation preferentially import and utilize the lactate produced by their neighbors (Hanahan and Weinberg, 2011 and references therein). In Fe-deficient plants the presence of similarly specialized cell subpopulations has not been observed yet. However, in plant roots different tissues, and thereby different "cell populations," are actually present. Under Fe deficiency it has been observed that the outer cell layers of the root are predominantly implicated in the activation of the typical enzymes (i.e., Ferric-chelate reductase, $\mathrm{H}^{+}$-ATPase) involved in the $\mathrm{Fe}$ uptake mechanism from the soil (Santi and Schmidt, 2008). Moreover the accumulation of the $\mathrm{H}^{+}$-ATPase protein was particularly high in the rhizodermal and endodermal cells compared to other layers in Fe-deficient cucumber roots (Dell'Orto et al., 2002). Thus, in Fe-deficient plants 
could the rhizodermal cells be considered as glucose-dependent cells whereas the cortex layers be considered as the second subpopulation of cells as observed in tumors? This question remains open until now, since no evidence about a different metabolic reprogramming between the different layers of the root are present: indeed all the studies carried out on the metabolic reprogramming in Fe-deficient plants have been performed on the total root system without discriminating between the different root tissues and layers.

Another important difference between Fe-deficient plant cells and animal tumor cells resides in the different use of glucose. Indeed, in animal tumor cells the Warburg effect is sustained by an increased uptake of glucose inside the proliferating cells (see above). In Fe-deficient plant cells the photosynthesis impairment leads to a lower glucose availability in the whole plant, this aspect being apparently in contrast with what is observed in tumor cells. On the other hand, in Fe-deficient plants, as long as glucose is available it seems to be directed to sustain the reprogrammed metabolism rather than biomass production, resulting in reduced plant growth, which is a general effect of Fe deficiency in plants.

One important aspect for the tumor biology in animal cells is represented by hypoxia. As was said before, hypoxia is important for the late-occurring events in developing cancer cells and it is not the direct cause of the Warburg effect. Thus it could be important to consider whether the hypoxia-inducing metabolic adaptation process is comparable in plant and animal cells. Similarly to what is observed in the animal counterpart, plant cells subjected to hypoxia show analogous metabolic effects (Licausi, 2011). Indeed, the most obvious effect of an oxygen shortage is an energy deficit. However, in plants, an energy deprivation is a common consequence of almost all abiotic stresses that negatively affect photosynthesis and/or respiration. Under these conditions, cells are still able to produce ATP via oxidative phosphorylation, but the rate of oxygen consumption is reduced and cells optimize energy usage by reorganizing metabolic fluxes. From this point of view, metabolic changes occurring under $\mathrm{Fe}$ deficiency are similar to those induced by hypoxia. Nevertheless, metabolic hypoxia-inducible changes, in plants, are mediated by an arsenal of transcription factors different from the HIF-P4DH system operating in cancer cells (Licausi et al., 2012).

Interestingly, HIF-1 cooperates with the c-myc gene to promote aerobic glycolysis (Dang et al., 2008). C-myc is a protooncogene that regulates the expression of several molecules involved in Fe homeostasis, including the Fe transporter DMT1 and frataxin. Hence, in animal cells HIF-1 effects are linked to Fe metabolism. Indeed, the high proliferation rate of tumor cells leads to hypoxia and/or mineral deficiencies in these cells. In fact, in response to hypoxia, the capacity of red blood cells to transport oxygen is up-regulated by the expression of genes involved in Fe metabolism: hypoxia up-regulates transferrin (Tf), which transports $\mathrm{Fe}^{3+}$ into cells, and ceruloplasmin (also known as ferroxidase), which is required to oxidize ferrous $\left(\mathrm{Fe}^{2+}\right)$ to ferric $\left(\mathrm{Fe}^{3+}\right)$ ions. Increasing expression of these genes supports Fe supply to the cell (Ke and Costa, 2006). Studies carried out by Callapina et al. (2005) have shown that decreased Fe levels are also able to mimic the hypoxia condition. Since Fe is required for the enzymatic activity of prolyl hydroxylase, depletion of intracellular Fe results in inactivation of the enzyme. The inactive PHD is unable to hydroxylate HIF- $1 \alpha$ and thus its subsequent degradation does not occur, leading to the transcription of HIF-1 target genes. Hence, in animal cells, Fe deficiency leads to an impairment of mitochondria together with an increase of both glycolysis and lactate production (Finch et al., 1979). Taking together all these findings, it is clear that $\mathrm{Fe}$ deficiency induces similar metabolic adaptations both in plant and in animal cells.

The understanding of Fe homeostasis in plants has increased tremendously in recent years. Considerable progress has been made during the last few years in understanding the importance of bHLH proteins, such as FIT, BHLH038, bHLH039, and PYE, as transcriptional regulators of Fe deficiency responses (Ivanov et al., 2012).

But, what about the regulation of the complex metabolic reprogramming? Are the metabolic changes in Fe-deficient plants regulated in the same manner as the metabolic changes occurring in tumor cells? Several PHD-like isoforms (e.g., $\mathrm{P} 4 \mathrm{Hs}$ ) have been cloned and characterized in plants (Vlad et al., 2007a). Although there is no evidence about the presence of HIF-1 homologs in plants, the possibility that P4Hs plays a role similar to that of HIF$\mathrm{P} 4 \mathrm{Hs}$ in response to abiotic stresses cannot be ruled out (Vlad et al., 2007b). Indeed, a recombinant Arabidopsis P4H (At-P4H-1) was found to hydroxylate synthetic peptides representing the two hydroxylated sequences in animal transcription factor HIF- $1 \alpha$, indicating substrate specificity for a transcription factor from a heterologous organism (Hieta and Myllyharju, 2002). However, even if there is no clear evidence for the presence of an HIF-1-P4H-like system in plants, indirect evidence does not allow us to exclude the presence of such proteins in plants.

In summary, I have shown here interesting similarities between plants and animals in order to prompt an open debate on the reinterpretation of the metabolic reprogramming of Fe-deficient plant cells as a Warburg-like effect. The metabolic changes occurring in tumor cells are considered as a hallmark of cancer and its comprehension leads to the characterization of the Achilles' Heel of the tumor, stimulating the identification of an arsenal of therapeutic compounds to use during chemotherapy. From this point of view, could the metabolic reprogramming occurring under Fe deficiency in plants be considered as a hallmark of the adaptation mechanism developed by plants to overcome the nutritional disorder?

Overall, it would be interesting to focus research toward the regulation of metabolic adaptation processes as well, in order to identify other candidate genes able to determine tolerance to Fe deficiency in plants.

\section{ACKNOWLEDGMENTS}

I wish to thank Prof. Graziano Zocchi and Dr. Marta Dell'Orto for critically reading the manuscript. I also wish to thank the Editor and the reviewers for their precious comments to improve the manuscript. The author was supported by "Dote Ricerca": FSE, Regione Lombardia.

\section{REFERENCES}

Brahimi-Horn, M. C., Chiche, J., and Pouyessegur, J. (2007). Hypoxia signalling controls metabolic demand. Curr. Opin. Cell Biol. 19, 223-229.

Callapina, M., Zhou, J., Schnitzer, S., Metzen, E., Lohr, C., Deitmer, J. W., and Brune, B. (2005). Nitric oxide reverses desferrioxamine- and hypoxia-evoked HIF-1 alfa accumulation -implication for prolyl hydroxylase activity and iron. Exp. Cell Res. 306, 274-284. 
Carew, J. S., and Huang, P. (2002). Mitochondrial defects in cancer. BMC Mol. Can. 1, 9. doi: 10.1186/1476-4598-1-9

Dang, C. V., Kin, J. W., Gao, P., and Yustain, J. (2008). The interplay between MYC and HIF in cancer. Nat. Rev. Cancer 8, 51-56.

Dell'Orto, M., Pirovano, L., Villalba, J. M., GonzalezReyes, J.A., and Zocchi, G. (2002). Localization of the plasma membrane H+-ATPase in Fe-deficient cucumber roots by immunodetection. Plant Soil 241, 11-17.

Donnini, S., Prinsi, B., Negri,A.S., Vigani, G., Espen, L., and Zocchi, G. (2010). Proteomic characterization of iron deficiency responses in Cucumis sativus L. roots. BMC Plant Biol. 10, 268. doi: 10.1186/1471-2229-10-268

Finch, C. A., Collnick, P. D., Hlastala, M. P., Miller, L. R., Dillmann, E., and Mackler, B. (1979). Lactic acidosis as a result of iron deficiency. J. Clin. Invest. 64 129-137.

Fischer, K., Hoffmann, P., Voelkl, S., Meidenbauer, N., Ammer, J., Edinger, M., Gottfried, E., Schwarz, S., Rothe, G., Hoves, S., Renner, K., Timischl, B., Mackensen, A., Kunz-Schughart, L., Andreesen, R., Krause, S. W., and Kreutz, M. (2007). Inhibitory effect of tumour cell-derived lactic acid on animal T cells. Blood 109, 3812-3819.

Gatenby, R. A., and Gillies, I. P. (2004). Why do cancers have high aerobic glycolysis? Nat. Rev. Cancer 4, 891-899.

Hanahan, D., and Weinberg, R. A. (2011). Hallmarks of cancer: the next generation. Cell 144, 646-674.

Harris, A. L. (2002). Hypoxia - a key regulatory factor in tumour growth. Nat. Rev. Cancer 2, 38-47.

Hieta, R., and Myllyharju, J. (2002). Cloning and characterization of a low molecular weight prolyl 4-hydroxylase from Arabidopsis thaliana. Effective hydroxylation of proline-rich, collagen-like and hypoxia-inducible transcription factor alpha-like peptides. J. Biol. Chem. 277, 23965-23971.

Ivanov, R., Brumbarova, T., and Bauer, P. (2012). Fitting into the harsh reality: regulation of iron-deficiency responses in dicotyledonous plants. Mol Plant 5, 27-42.
Ke, Q., and Costa, M. (2006). Hypoxia-inducible factor-1 (HIF-1). Mol. Pharm. 70, 1469-1480.

Koukourakis, M. I., Giatromanolaki, A., Harris, A. L., and Sivridis, E. (2006). Comparison of metabolic pathways between cancer cells and stromal cells in colorectal carcinomas: a metabolic survival role for tumor-associated stroma. Cancer Res. 66, 632-637.

Licausi, F. (2011). Regulation of the molecular response to oxygen limitations in plants. New Phytol. 190, 550-555.

Licausi, F., Kosmacz, M., Weits, D. A., Giuntoli, B., Giorgi, F. M., Voesenek, L. A., Perata, P., and van Dongen, J. T. (2012). Oxygen sensing in plants is mediated by an $\mathrm{N}$-end rule pathway for protein destabilization. Nature 479, 419-422.

López-Millán, A. F., Morales, F., Andaluz, S., Gogorcena, Y., Abadía, A., de Las Rivas, J., and Abadía, J. (2000). Responses of sugar beet roots to iron deficiency. Changes in carbon assimilation and oxygen use. Plant Physiol. 124, 885-897.

López-Millán, A. F., Morales, F., Gogorcena, Y., Abadia, A., and Abadia, J. (2009). Metabolic responses in iron deficienct tomato plants. J. Plant Physiol. 166, 375-384.

Rellán-Álvarez, R., Andaluz, S., Rodríguez-Celma, J., Wohlgemuth, G., Zocchi, G., Alvarez-Fernández, A., Fiehn, O., López-Millán, A. F., and Abadía, J. (2010). Changes in the proteomic and metabolic profiles of Beta vulgaris root tips in response to iron deficiency and resupply. BMC Plant Biol. 10, 120. doi: 10.1186/1471-2229-10-120

Santi, S., and Schmidt, W. (2008). Laser microdissection-assisted analysis of the functional fate of iron deficiency-induced root hairs in cucumber. J. Exp. Bot. 59,697-704.

Semenza, G. L. (2007). Hypoxia-inducible factor 1 (HIF1) pathway. Science STKE. 2007, cm8.

Swietach, P., Vaughan-Jones, R. D., and Harris, A. L. (2007). Regulation of tumour $\mathrm{pH}$ and the role of Carbonic anhydrase 9. Cancer Metastasis Rev. 26, 299-310.
Taylor, C. T., and Pouyssegur, J. (2007). Oxygen, hypoxia and stress. Ann. N. Y. Acad. Sci. 1113, 87-94.

Thimm, O., Essigmann, B., Kloska, S., Altmann, T., and Buckhout, T.J. (2001). Response of arabidopsis to iron deficiency stress as revealed by microarray analysis. Plant Physiol. 127, 1030-1043.

Vander Heiden, M. G., Cantley, L. C., and Thompson, C. B. (2009). Understanding the Warburg effect: the metabolic requirements of cell proliferation. Science 324, 1029-1033.

Vigani, G. (2012). Discovering the role of mitochondria in the iron deficiency-induced metabolic responses of plants. J. Plant Physiol. 168, 1-11.

Vlad, F., Spano, T., Vlad, D., Buo Daher, F., Ouelhadj, A., and Kalaitzis, P. (2007a). Arabidopsis prolyl 4-hydroxylases are differentially expressed in response to hypoxia, anoxia and mechanical wounding. Physiol Plant 130, 471-483.

Vlad, F., Spano, T., Vlad, D., Buo Daher, F., Ouelhadj, A., Fragkostefanakis, S., and Kalaitzis, P. (2007b). Involvement of Arabidopsis prolyl 4 hydroxylases in hypoxia, anoxia and mechanical wounding. Plant Signal Behav. 2, 638-369.

Warburg, O. (1956). On the origin of cancer cells. Science $123,309-314$.

Warburg, O., Posener, K., and Negelein, E. (1924). Uber den Stoffwechsel der Tumoren. Biochem. Zeit. 152,319-344.

Received: 06 February 2012; accepted: 27 February 2012; published online: 13 March 2012.

Citation: Vigani G (2012) Does a similar metabolic reprogramming occur in Fe-deficient plant cells and animal tumour cells? Front. Plant Sci. 3:47. doi: 10.3389/ fpls.2012.00047

This article was submitted to Frontiers in Plant Nutrition, a specialty of Frontiers in Plant Science.

Copyright (c) 2012 Vigani. This is an open-access article distributed under the terms of the Creative Commons Attribution Non Commercial License, which permits noncommercial use, distribution, and reproduction in other forums, provided the original authors and source are credited. 